\title{
The Research on Microstructure and Properties of WQ960 Welded Joints under Longitudinal Magnetic Field
}

\author{
Lu Hailong \\ Jilin vocational college of industry and technology \\ jilin, china \\ e-mail: 289646841@qq.com \\ Wang Lixiang \\ AVIC liming jinxi chemical machinery Co.,Ltd, \\ huloudao, china \\ e-mail: 289646841@qq.com
}

\author{
Su Yunhai \\ shenyang university of technology \\ Shenyang, china \\ e-mail: 289646841@qq.com
}

\begin{abstract}
The WQ960 steel is high strength steel, which is widely used. The WQ960 high strength steel was welded by gas metal arc welding under longitudinal magnetic field. The tensile strength, impact properties and microstructure were tested by tensile test, impact test and metallograghy test, which were under different processing parameters. The influence and acting mechanism of magnetic field on the microstructure, impact toughness and tensile strength of the welded joints were researched. The results show that magnetic field can improve the tensile strength, impact energy and microstructure of welded joints. Under the effect of magnetic field, the dimples in the fiber area of impact fracture of welded joints were deeper and more uniform. When the excitation current is $2.5 \mathrm{~A}$, the magnetic field frequency is $20 \mathrm{~Hz}$, the tensile strength of welded joints and low impact energy reach the maximum, respectively $839 \mathrm{MPa}$ and $71 \mathrm{~J}$ increased $17.01 \%$ and $22.41 \%$. The longitudinal magnetic field is applied to improve the weld crystalline form, reduce the number of PF and FSP, and form more AF.
\end{abstract}

Keywords-HSLA; Magnetic field; grain refinement; mechanical property

\section{INTRODUCTION}

According to the development of science and engineering, the demand of material is more and more high. Because of its high strength, good plasticity and ductility, the low alloy high strength steel is widely used, such as engineering machinery, bridge and shipping industry, and so on. The high strength steel is always the fine grain steel. The more the grain is fine, the more the strength is high. For the process of welding, the material of welded joint melts and solidifies fastly, so the growth of grain will be changed, the microstructure and mechanical properties of high strength steel welded joints will change obviously. This will seriously limit the application of high strength steel. Therefore, it is necessary to study the relevant problems of the low alloy high strength steel in the welding process. Lots of studies have shown that magnetic controlling welding can better control the quality of welded joints than other traditional welding method, which is regarded as "nondestructive welding". The external magnetic field can refine grain, reduce chemical inhomogeneity and the sensitivity of the crystal cracks and pores, and increase the plasticity and toughness of welded joint. So this method was used to improve the properties of weld metal, comprehensively improve the quality of the welded joints. Due to the simple additional device, low cost, high efficiency, environmental protection and other advantages, electromagnetic stirring welding technique is widely used in magnesium alloy, aluminum alloy or other mental. So, the magnetic field is used when the low alloy high strength steel is welded by GMAW. Through the act of magnetic field, the properties of welded joint may be improved. This will get new way for the welding of low alloy high strength steel.

\section{TEST MATERIAL AND METHOD}

The base metal is WQ960 plate with the dimension of $200 \mathrm{~mm} \times 60 \mathrm{~mm} \times 10 \mathrm{~mm}$. According to the base metal compositions (shown as table 1)and the requirements of HSLA steel welding, the JM100 welding wire was elected. Wire diameter(shown as table 2) is $\varphi 1.2 \mathrm{~mm}$ and the tensile strength is up to $715 \mathrm{MPa}$. The welding current is $220 \mathrm{~A}$ and the voltage is $24 \mathrm{~V}$. The chemical composition of the base metal and the wire are showed in Table1 and Table2. The joint form is $\mathrm{V}$-shaped groove with the bevel angle of $60^{\circ}$; blunt edge of $2 \mathrm{~mm}$ and plate spacing of $2 \mathrm{~mm}$. The rust on the grooves is cleared before welding.

TABLEI CHEMICAL COMPOSITION OF EXPERIMENTAL MATERIALS

$\begin{array}{llllllllllll}\text { C } & \text { Si } & \text { Mn } & \text { S } & \text { P } & \text { Cr } & \text { Ni } & \text { Mo } & \text { Nb } & \text { V } & \text { Ti } & \text { B }\end{array}$

$\begin{array}{llllllllllll}0.18 & 0.50 & 1.60 & 0.01 & 0.02 & 0.08 & 1.00 & 0.60 & 0.05 & 0.05 & 0.03 & 0.005\end{array}$

TABLEII CHEMICAL COMPOSITION OF WELDING WIRE

$\begin{array}{lllllllllll}\mathrm{C} & \mathrm{Si} & \mathrm{Mn} & \mathrm{Cu} & \mathrm{P} & \mathrm{Ni} & \mathrm{Mo} & \mathrm{Ti} & \mathrm{Al} & \mathrm{Fe}\end{array}$

$\begin{array}{llllllllll}0.07 & 0.58 & 1.61 & 0.10 & 0.016 & 0.84 & 0.39 & 0.092 & 0.02 & \text { other }\end{array}$ 
The mental was welded by MAG under Longitudinal Magnetic Field (LMF-MAG). Fig .1 is the schematic diagram of LMT-MAG. The LMF-MAG is mainly composed of magnetic field power and excitation coil which is fixed on the welding gun. During the welding process, the magnetic field current and frequency can be changed.

After welding, tensile strength and impact toughness of welded joints were tested, and the microstructure of welded mental was observed and analyzed by OLNMPUS BX-6 metallographic microscope.

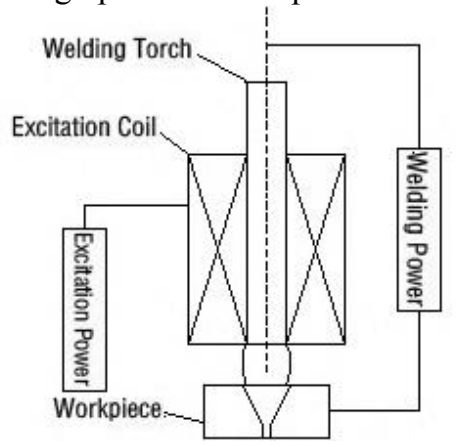

Figure 1. Schematic diagram of LMF-MAG

\section{TEST RESULTS AND ANALYSIS}

Fig .2 is the microstructure of weld mental under different magnetic field. From the Fig .2, it can be found that the microstructure of weld mental is changed under different magnetic field parameters. Without magnetic field, the microstructure of weld metal mainly consists of acicular ferrite (AF), proeutectoid ferrite (PF) and ferrite side-plate (FSP). The PF precipitates along the austenite grain boundaries (AGB), which are completely covered, FSP grows from AGB to the grain, and the majority of microscopic structure in the austenitic grain is intertwined $\mathrm{AF}$, shown as Fig .2 a. When the magnetic field is used and the parameters are suitable, the microstructure is best. The number of AF in weld metal increases significantly, only a few PF discontinuously distributes along the austenite grain boundaries, the number and size of FSP is also significantly reduced, shown as Fig $.2 \mathrm{~b}$. When the magnetic field parameters are increasing continuously, the PF almost disappears in the welded mental, the number and size of FSP increases relative to Fig .2 b, shown as Fig $.2 \mathrm{c}$.

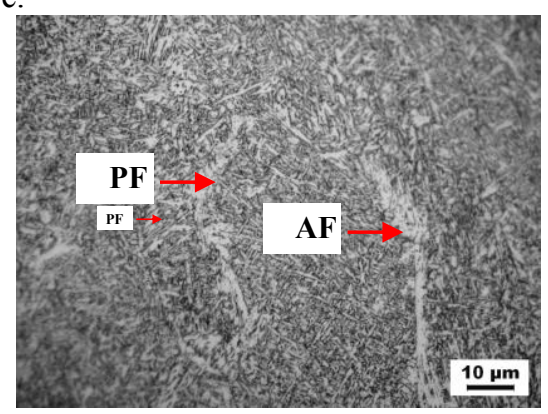

(a) $\operatorname{Im}=0 \mathrm{~A}, \mathrm{f}=0 \mathrm{~Hz}$

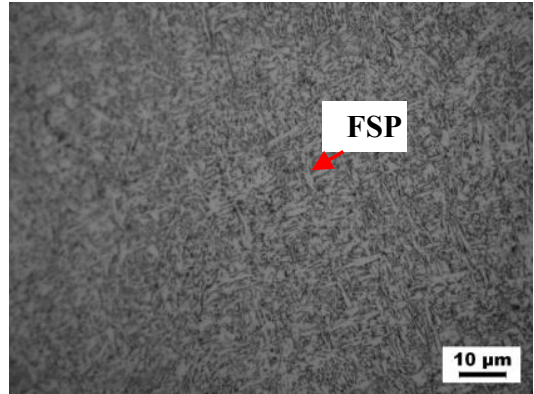

(b) $\operatorname{Im}=2 \mathrm{~A}, \mathrm{f}=20 \mathrm{~Hz}$

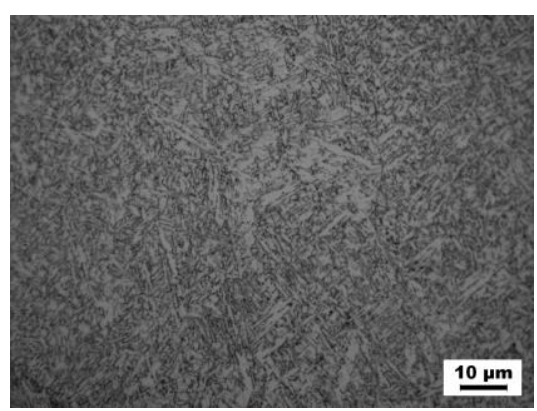

(c) $\operatorname{Im}=2.5 \mathrm{~A}, \mathrm{f}=25 \mathrm{~Hz}$

Figure 2. Microstructure of weld metal

In summary, the number of PF and FSP in weld mental under longitudinal magnetic field reduces obviously relative to traditional welded joints, which means that magnetic field can refine the grain. However, magnetic field doesn't always improve the microstructure, if the magnetic field parameter is inappropriate, the grain of welded joints will appear coarsening trend.

The tensile strength and low temperature $\left(-20{ }^{\circ} \mathrm{C}\right)$ impact energy of welded joints without longitudinal magnetic field are $717 \mathrm{MPa}$ and 58J. Fig .3 and 4 are tensile strength and impact absorbing energy of welded joint under different magnetic field parameter. The figure shows that when the field current is less than $2.5 \mathrm{~A}$, the magnetic field frequency is less than $20 \mathrm{~Hz}$, the tensile strength and low impact energy of welded joint are improving with the increase of the field current and frequency, when the field current and frequency respectively reaches $2.5 \mathrm{~A}$ and $20 \mathrm{~Hz}$, the tensile strength and low impact energy of welded joints reach the maximum, respectively $839 \mathrm{MPa}$ and $71 \mathrm{~J}$, increasing $17.01 \%$ and $22.41 \%$; If the current and frequency of the magnetic field continue to increase, the tensile strength and low impact energy of welded joints decrease.

According to microstructure, the development of tensile strength of HSLA welded joints in the appropriate magnetic field is because the grain is refined. With the increasing of the field current and frequency, the grain of welded joints coarsens, thus the tensile strength decreases. The number, size and distribution of inclusions in welded joint will be changed by magnetic field, but the data(Fig .2)shows that inclusion does not have obvious impact on the tensile strength of material. 


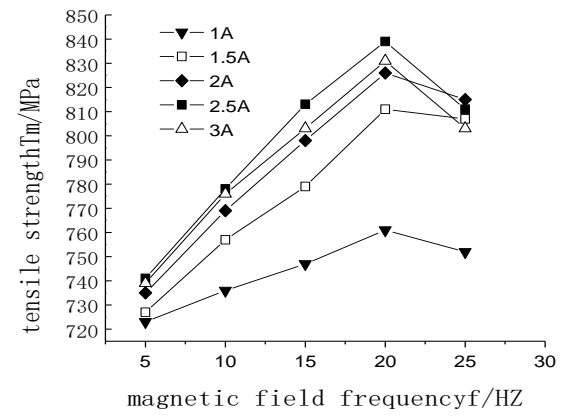

Figure 3. Tensile strength of welding joint under different magnetic field frequency

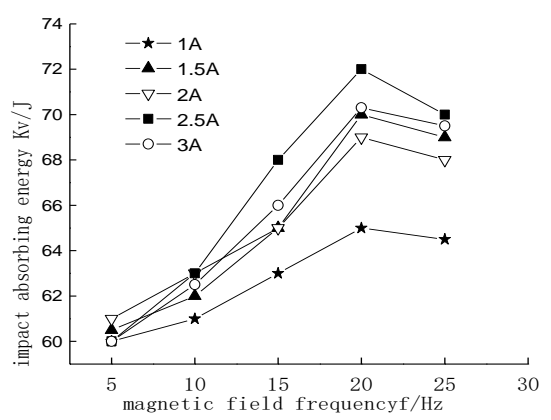

Figure 4. Impact absorbing energy of welding joint under different magnetic field frequency

It's complex that the influence of magnetic field on the low temperature impact toughness, so we need to combine the fractograph for further analysis. Impact fractograph usually consists of fiber areas, radiation areas, and cut lip. Radiation fracture zone is brittle fracture, the energy consumption of which is very small relative to plastic fiber fracture zone; because the crack propagation energy is mainly consumed in the dimples formed on the plastic deformation [6], so this paper mainly analyzes fiber area .Fig .4 is low-temperature impact fracture SEM image of HSLA welded joints. In Fig .4, fractograph is typical equiaxed dimples; there are inclusions or pores at the bottom of dimples, which indicate that the mechanism of the dimple is microporous coalescence fracture. In Fig .4a, we can see the dimples are shallow and small; but in Fig .4b, the number of large dimples significantly increases and dimple depth also increases.

According to the microstructure, the change shown as above-mentioned is because that the number of AF in the weld mental increases obviously, and the growth of PF is suppressed. Cracks expand fast at PF, which means that the energy consumption is relatively small, so the dimples are small and shallow. However, the distribution form of $\mathrm{AF}$ is high-angle boundary, forming interwoven structure. So the path of crack propagation increases, which means the stronger resistance to crack growth, the larger energy consume to plastic deformation, resulting in large and deep dimples [7]。
Longitudinal magnetic field affects the performance of the organization and properties of welded joints from three main aspects : the cooling rate, inclusions and physical agitation [8]。

According to Bhadeshia[9], PF grows in the planar manner after nucleation along $\mathrm{AGB}$, and the growth rate is determined by the diffusion rate of carbon in leading edge of austenite. In the longitudinal magnetic field, the rotation of arc decreases the weld current[10]。 The instantaneous cooling rate of weld metal [11] is :

$$
\omega_{\mathrm{c}=f(\varepsilon)} \frac{2 \pi \lambda(\mathrm{T}-\mathrm{T} 0)^{2} v}{\eta I U}
$$

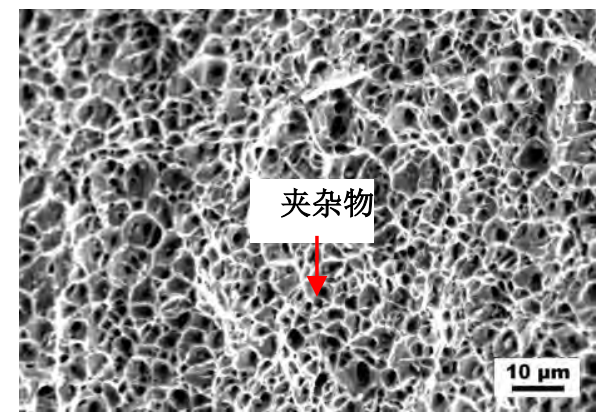

(a) $\operatorname{Im}=0 \mathrm{~A}, \mathrm{f}=0 \mathrm{~Hz}$

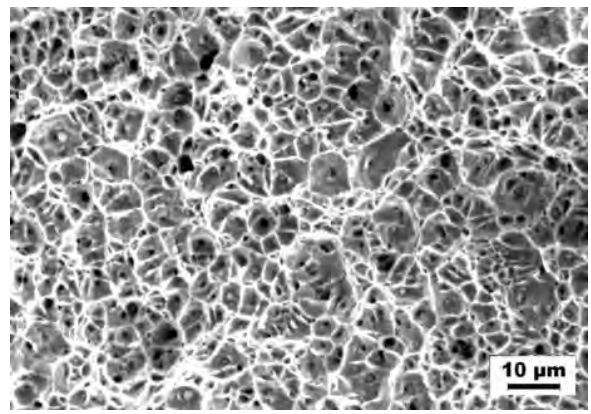

(b) $\mathrm{Im}=2 \mathrm{~A}, \mathrm{f}=2 \mathrm{~Hz}$

Figure 5. SEM fractographs of Charpy specimens

Wherein, $\omega_{\mathrm{c}}$ is the cooling rate when the instantaneous temperature is $\mathrm{T} ; f(\varepsilon)$ is correction factor; T0 is preheat temperature; $v$ is welding speed; $\mathrm{I}$ is welding current; $\mathrm{U}$ is welding voltage; $\eta$ is thermal efficiency; $\lambda$ is thermal conductivity. In this experiment, $\mathrm{I}$ and $\mathrm{U}$ are constant, and the environment is relatively stable, so $f(\varepsilon)$ can be regarded as constant.

With the increasing of magnetic field current and frequency, the actual welding current is reduced, the cooling rate increases. On the other hand, the electromagnetic stirring accelerates the dissipation of heat, which further accelerate the cooling rate, since carbon is too late to spread outside AGB, the growth of PF is inhibited. However, if the magnetic field current and the frequency continue to increase, the actual current density will be smaller and heat dissipation will be faster, cooling speed is further increased. Too fast cooling rate would 
promote the growth of FSP, and the grains coarsen. Fig .1b and $\mathrm{c}$ demonstrate the changes in the analysis.

The nucleation of $\mathrm{AF}$ is belong on inclusions or $\mathrm{AF}$ that has been formed (induced nucleation). But not all the inclusions can become acicular ferrite nucleation foundation, only inclusions in $0.2 \sim 0.6 \mu \mathrm{m}$ size are in favor of AF nucleation, Oversized inclusions can reduce welded joints toughness, in the impact test, the hole in dimple bottom are formed generally first near the large-sized inclusions, thereby reduce the impact toughness [12]. Under electromagnetic stirring, the agitation of molten bath increases the chance of the molten bath contacting with $\mathrm{CO} 2$ in shielding gas. This will promote the formation of oxides so that increase the number of inclusions. Molten bath will form a rotary motion and aggravate the convection of metal bath. According to ostwald theory, the big inclusions will devour the little by collision to form larger inclusions. During to the density of inclusions is much lower than the density of the liquid metal, inclusions tend to float to the surface of weld mental and be removed as slag. Regarding the metal bath as ideal liquid, according to Stockes law, the rising speed $v$ of inclusions is:

$$
v=\frac{2\left(\rho_{M}-\rho_{z}\right) g r^{2}}{9 \eta_{M}}
$$

Wherein, $\rho M$ is liquid metal density, $\rho z$ is inclusions density, $r$ is the radius of the inclusions; $\eta \mathrm{m}$ is the viscosity of the molten pool; $\mathrm{g}$ is the gravity acceleration. According to equation (2).

we know that larger size of inclusions is, the lest number of inclusion is. On the other hand, since the metal bath will produce vigorous stirring by the Lorentz force, and mechanical stirring will also help break the large grains, increase the nucleation rate, and refine microstructure.

\section{CONCLUSION}

(1) When the excitation current to $2.5 \mathrm{~A}$, the magnetic field frequency to $20 \mathrm{~Hz}$, the tensile strength of welded joints and low impact energy reach the maximum, respectively $839 \mathrm{MPa}$ and $71 \mathrm{~J}$ increased $17.01 \%$ and $22.41 \%$.

(2)The longitudinal magnetic field is applied to improve the weld crystalline form, reduce the number of $\mathrm{PF}$ and FSP, and form more AF.

(3)Applying the magnetic field, the dimples in fiber area are more uniform and generally darker than these without magnetic field in the low-temperature impact experiments.

\section{REFERENCES}

[1] Jiang Shuyuan. Chen Huanming. Application and progress of magnetron technology in welding $[\mathrm{J}]$. China Mechanical Engineering, 2002, 13(21): 1876-1879.

[2] Jia Changshen. Yin Xianqing. The welding arc behavior in longitudinal magnetic field[J]. Academic Journal of Xi'an Jiaotong University, 1994, 28(4): 7-13.

[3] Su Yunhai. Jiang Huanwen. Wu Deguang. Optimal design of magnesium alloy magnetron TIG welding process parameters $[\mathrm{J}]$ Welding Institution, 2012, 33(012): 85-88.

[4] Su Yunhai. Liu Zhenjun. Wang Yu. The influence of magnetic field parameters on the microstructure and mechanical properties of AZ31 welded joint [J]. Welding Institution, 2007, 28(5): 45-48.

[5] Xia Zhixin. Yang Zuoyue. Su Jie. The influence of the type and shape of inclusions on the plastic properties of ultra high strength[J]. Journal of Aeronautical Materials, 2008, 28(5): 17-17.

[6] Li Yuqing. Guan Yun. The austenitic grain boundary $\mathrm{Cr} 2 \mathrm{~N}$ of heat resistant steel $5 \mathrm{Cr} 21 \mathrm{Mn} 9 \mathrm{Ni} 4 \mathrm{~N}[\mathrm{~J}]$. Electron Microscopy Society, 1998, 17(1): 33-38.

[7] Jiang Qinglei. Investigation of microstructure, properties and fine structure of 800MPA high strength steel joints by GMAW[D] Shandong university, 2011.

[8] Huang Guoan Yu Shengfu. Acicular ferrite microstructure of lowalloy steel weld [J]. Welding Institution.2008,29(3):45-48.

[9] Villafuerte J C, Kerr H W. Electromagnetic stirring and grain refinement in stainless steel GTA welds[J]. 1990.

[10] Chen Shujun. Hua Aibing.. Arc movement characteristics of TIG welding in a rotating magnetic field[J]. Weld, 2006 (10): 34-36.

[11] Zhang Wenyue. Welding heat transfer[M]. Machinery Industry Press, 1989:86.

[12] Leslie W C. The physical metallurgy of steels[M]. Hempisphere Publishing Corporation, 1981:269. 